\title{
The K2K SciBar Detector
}

K. Nitta ${ }^{\text {a }}$, E. Aliu ${ }^{\text {b }}$ S. Andringa ${ }^{\text {b }}$, S. Aoki ${ }^{\text {, }}$, S. Choi ${ }^{\mathrm{d}}$, U. Dore $^{\mathrm{e}}$, X. Espinal ${ }^{\mathrm{b}}$, J. J. Gomez-Cadenas ${ }^{\mathrm{f}}$, R. Gran ${ }^{\mathrm{g}}$, M. Hasegawa ${ }^{\text {h }}$ K. Hayashi ${ }^{\text {h }}$, K. Hayashi ${ }^{i}$, Y. Hayato ${ }^{\text {a }}$, K. Hiraide ${ }^{\mathrm{h}}$, A. K. Ichikawa ${ }^{\mathrm{a}}$, M. Iinuma ${ }^{\mathrm{i}}$, J. S. Jang ${ }^{\mathrm{j}}$, E. J. Jeon ${ }^{\mathrm{d}}$, K. K. Joo ${ }^{\mathrm{d}}$, C. K. Jung ${ }^{\mathrm{k}}$, I. Kato ${ }^{\mathrm{h}}$, D. Kerr ${ }^{\mathrm{k}}$, J. Y. Kim ${ }^{\mathrm{j}}$, S. B. Kim ${ }^{\mathrm{d}}$, K. Kobayashi ${ }^{\mathrm{k}}$, A. Kohara ${ }^{\mathrm{i}}$, J. Kubota $^{\text {h}}$, Yu. Kudenko ${ }^{\ell}$, Y. Kuno ${ }^{\mathrm{m}}$, M. J. Lee ${ }^{\mathrm{j}}$, E. Lessac-Chenin ${ }^{\mathrm{k}}$, I. T. Lim ${ }^{\mathrm{j}}$, P. F. Loverre ${ }^{\mathrm{e}}$, L. Ludovici ${ }^{\mathrm{e}}$, H. Maesaka ${ }^{\text {h }}$ C. Mariani ${ }^{\text {e }}$, C. McGrew ${ }^{\mathrm{k}}, \mathrm{O}_{\text {Mineev }}{ }^{\ell}$, T. Morita ${ }^{\text {h }}$, T. Murakami ${ }^{\text {a }}$, Y. Nakanishi ${ }^{i}$, T. Nakaya ${ }^{\text {h }}$, S. Nawang ${ }^{\text {, }}$ K. Nishikawa ${ }^{\text {h }}$, M. Y. Pac ${ }^{n}$, E. J. Rhee ${ }^{\text {, }}$,

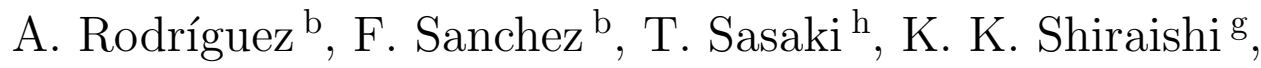
A. Suzuki ${ }^{c}$, T. Takahashi ${ }^{\text {i }}$, Y. Takubo ${ }^{\mathrm{m}}$, M. Tanaka ${ }^{\mathrm{a}}$, R. Terri ${ }^{\mathrm{k}}$, A. Tornero-Lopez ${ }^{\mathrm{f}}$, S. Ueda ${ }^{\mathrm{h}}$, R. J. Wilkes ${ }^{\mathrm{g}}$, S. Yamamoto ${ }^{h}$, M. Yokoyama $^{\mathrm{h}}$, and M. Yoshida ${ }^{\mathrm{m}}$

${ }^{a}$ Institute of Particle and Nuclear Studies, KEK, Tsukuba, Ibaraki 305-0801, JAPAN

b Universitat Autónoma de Barcelona Institut de Física d'Altes Energies Edifici Cn, E-08193, Barcelona, SPAIN

${ }^{\mathrm{c}}$ Kobe University, Kobe, Hyogo 657-8501, JAPAN

${ }^{\mathrm{d}}$ Department of Physics, Seoul National University, Seoul 151-742, KOREA

"Universita' di Roma "La Sapienza" and INFN Roma, ITALY

${ }^{\mathrm{f}}$ IFIC - Instituto de Fisica Corpuscular Apartado de Correos 22085 E-46071 Valencia, SPAIN

g Department of Physics, University of Washington, Seattle, WA 98195-1560, USA

${ }^{\mathrm{h}}$ Department of Physics, Kyoto University, Kyoto 606-8502, JAPAN

${ }^{\mathrm{i}}$ Graduate School of Advanced Sciences of Matter, Hiroshima University, Higashi-Hiroshima, 739-8530, JAPAN

jDepartment of Physics, Chonnam National University, Kwangju 500-757, KOREA

${ }^{\mathrm{k}}$ Department of Physics and Astronomy, State University of New York, Stony Brook, NY 11794-3800, USA

${ }^{\ell}$ Institute for Nuclear Research RAS, 117312 Moscow, RUSSIA 
${ }^{\mathrm{m}}$ Department of Physics, Osaka University, Toyonaka, Osaka 560-0043, JAPAN

${ }^{\mathrm{n}}$ Department of Physics, Dongshin University, Naju 520-714, KOREA

\begin{abstract}
A new near detector, SciBar, for the K2K long-baseline neutrino oscillation experiment was installed to improve the measurement of neutrino energy spectrum and to study neutrino interactions in the energy region around $1 \mathrm{GeV}$. SciBar is a 'fully active' tracking detector with fine segmentation consisting of plastic scintillator bars. The detector was constructed in summer 2003 and is taking data since October 2003. The basic design and initial performance is presented.
\end{abstract}

\title{
1 Introduction
}

The KEK-to-Kamioka long-baseline neutrino experiment (K2K)[1] started taking data in 1999. An almost pure muon neutrino beam with average energy of $1.3 \mathrm{GeV}$ is produced with the KEK $12-\mathrm{GeV}$ proton synchrotron and directed toward the Super-Kamiokande detector (SK) located at Kamioka, $250 \mathrm{~km}$ away from KEK. The neutrino flux and energy spectrum at SK is estimated from the flux measured by near detectors located $300 \mathrm{~m}$ downstream from the production target. The number of events and the spectral shape at SK are compared with the expectations to study neutrino oscillations. The latest $\mathrm{K} 2 \mathrm{~K}$ results[2] indicate neutrino oscillation, and are consistent with the $\mathrm{SK}$ results[3]. In $\mathrm{K} 2 \mathrm{~K}$, the neutrino energy at the oscillation maximum is expected to be $\sim 0.6 \mathrm{GeV}$.

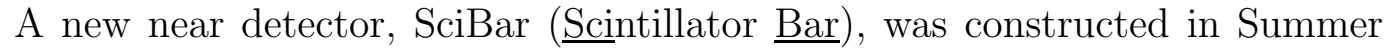
2003 to upgrade the near detectors. A main motivation of the new detector is to improve the measurement of neutrino energy spectrum by using Charged Current Quasi Elastic interaction (CCQE, $\nu_{\mu}+n \rightarrow \mu^{-}+p$ ). In order to select CCQE interaction with high purity and high efficiency and to suppress other interactions such as inelastic interactions with pions $\left(\nu_{\mu}+p \rightarrow \mu^{-}+p+\right.$ $\pi^{+}$), the detector is designed to have high efficiency for all charged particles produced in the interaction. The detector consists of plastic scintillator strips with fine segmentation. The scintillator itself is a neutrino target and has no dead region. Due to the fine segmentation of the detector, short tracks down to $10 \mathrm{~cm}$ long can be detected. The detector also has a capability of particle identification (especially for protons and pions) with $\mathrm{dE} / \mathrm{dx}$ information by measuring the energy deposit in each strip.

In addition, we expect to provide many new results for neutrino interactions in the $1 \mathrm{GeV}$ region with SciBar. 


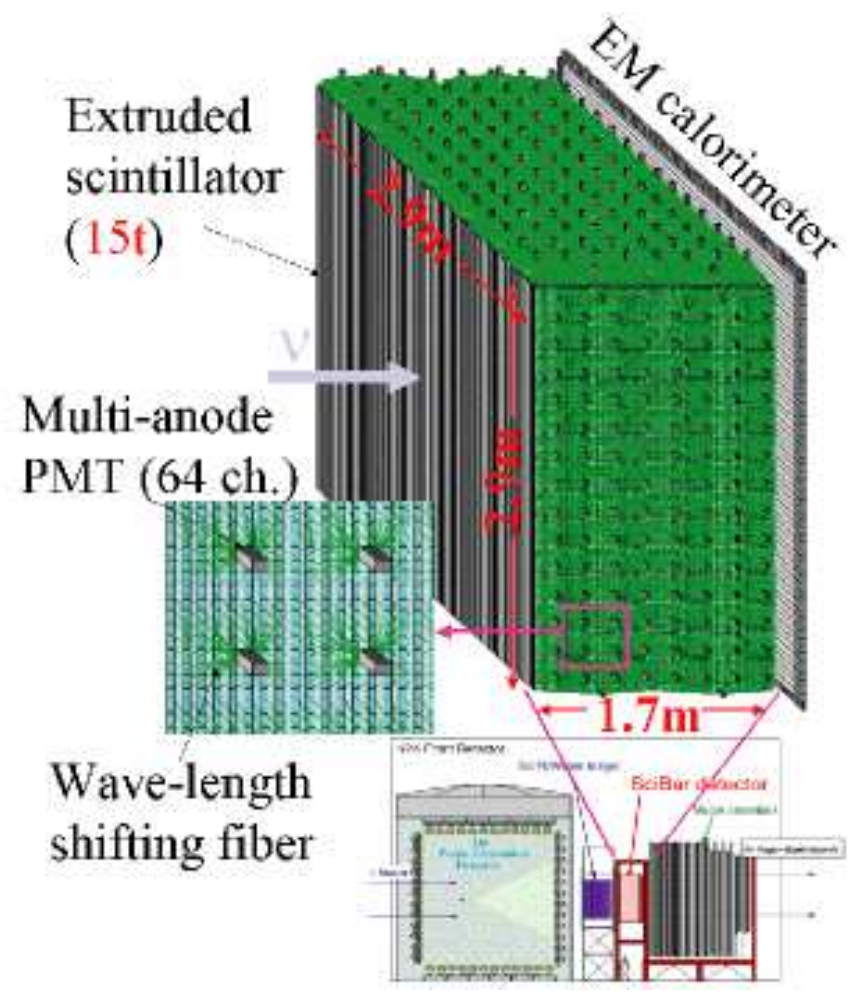

Fig. 1. Schematic drawings and description of the SciBar detector.

\section{The SciBar Detector}

Figure 1 shows a schematic view of SciBar. SciBar consists of two components: a tracking calorimeter made of scintillator strips and an electromagnetic calorimeter called Electron Catcher. Table 1 shows the design for each component. The SciBar tracker consists of 14848 extruded scintillator strips with each dimension of $1.3 \times 2.5 \times 300 \mathrm{~cm}^{3}$. The scintillator strips are arranged in 64 layers. Each layer consists of two planes, with 116 strips glued together to give horizontal and vertical position. The total size and weight are $2.9 \times 2.9 \times 1.7 \mathrm{~m}^{3}$ and 15 tons, respectively. Each strip is read out by a wavelength shifting (WLS) fiber attached to a 64-channel multi-anode PMT (MAPMT). Charge and timing information from MAPMT is recorded by custom designed electronics[4]. Two outermost strips in each horizontal and vertical planes are called as Outer Detector (OD). For the OD readout, eight 1-ch PMTs with WLS fibers are used to identify incoming and outgoing events.

The scintillator strips are made of polystyrene, infused with PPO (1\%) and POPOP $(0.03 \%)$, and are produced by extrusion in the shape of rectangular bar with $\mathrm{TiO}_{2}$ reflecting coating $(0.25 \mathrm{~mm}$ thickness). Scintillators are produced at FNAL and the composition of the material is the same as those used for the MINOS experiment[5]. Each scintillator has a $1.8 \mathrm{~mm}$ diameter hole where the $1.5 \mathrm{~mm} \phi$ WLS fiber is inserted for light collection. The fiber is 


\begin{tabular}{|c|c|c|}
\hline \multirow[t]{4}{*}{ Structure } & Dimensions & $2.9 \mathrm{~m}$ (horizontal), $2.9 \mathrm{~m}$ (vertical), $1.7 \mathrm{~m}$ (thick) \\
\hline & Weight & 15 tons \\
\hline & Number of Strips & 14,848 \\
\hline & Number of PMTs & $224(+\mathrm{OD}: 8)$ \\
\hline \multirow{5}{*}{$\begin{array}{l}\text { Scintillator } \\
\text { strip }\end{array}$} & Material & polystyrene, $\mathrm{PPO}(1 \%)$ and $\mathrm{POPOP}(0.03 \%)$ \\
\hline & Size & $1.3 \mathrm{~cm}$ (thick), $2.5 \mathrm{~cm}$ (wide), $3 \mathrm{~m}$ (long) \\
\hline & Weight & $1 \mathrm{~kg}$ \\
\hline & Coating & $0.25 \mathrm{~mm}\left(\mathrm{TiO}_{2}\right)$ \\
\hline & Emission length & $420 \mathrm{~nm}($ peak) \\
\hline \multirow[t]{4}{*}{ Fiber } & Diameter & $1.5 \mathrm{~mm} \phi$ \\
\hline & Reflective index & 1.59 (outer) / 1.50 (middle) / 1.42 (inner) \\
\hline & Absorption length & $430 \mathrm{~nm}$ (peak) \\
\hline & Emission length & $476 \mathrm{~nm}$ (peak) \\
\hline \multirow[t]{7}{*}{ PMT } & Model & H8804 \\
\hline & Cathode material & Bialkali \\
\hline & Anode & $8 \times 8\left(2 \times 2 \mathrm{~mm}^{2} /\right.$ pixel $)$ \\
\hline & Wave length sensitivity & 300-650nm (Max 420nm) \\
\hline & Number of dynode stage & 12 \\
\hline & Gain(@800V) & $3 \times 10^{5}$ \\
\hline & Quantum efficiency & $21 \%$ at $390 \mathrm{~nm}$ \\
\hline \multirow[t]{4}{*}{ DAQ } & Shaping time & $80 \mathrm{nsec}(\mathrm{TA}), 1.2 \mu \mathrm{sec}(\mathrm{VA})$ \\
\hline & Pedestal width & $0.03 \mathrm{MeV}$ \\
\hline & Linearity & $5 \%$ at $30 \mathrm{MeV}$ \\
\hline & Dynamic range & $0.1-30 \mathrm{MeV}$ \\
\hline
\end{tabular}

Table 1

Specification of each component of SciBar.

multi-clad type, Y11(200)MS, made by Kuraray. The attenuation lengths of all WLS fibers were measured before the installation. Their average is $350 \mathrm{~cm}$.

MAPMT is H8804 made by Hamamatsu Photonics K.K.. It's Anodes are arranged in an $8 \times 8$ array with each anode measuring $2 \mathrm{~mm} \times 2 \mathrm{~mm}$. The sensitive wave length is from $300 \mathrm{~nm}$ to $650 \mathrm{~nm}$, which matches the emission spectrum of the WLS fibers. The cross talk value in our configuration is less than $4 \%$. The gains of all channels have been measured before installation. The linearity is also measured to be $10 \%$ upto 300 photo-electrons (p.e.) with the gain of $6.3 \times 10^{5}$.

Readout system consists of frontend electronics attached to MAPMT and a backend VME module. A combination of ASICs (VA32HDR11 and TA32CG made by IDEAS) is used on the frontend readout electronics. VA is a 32-ch preamplifier chip with shaper and multiplexer. TA provides timing information 
after taking OR of 32 channels. Two packages of VA/TA are mounted on a custom-designed PCB board (Front-End board, FEB) to read out signals from 64 anodes. A backend electronics board (DAQB) has been also newly developed as a standard VME-9U board. DAQB controls and reads out eight FEBs. The charge information from MAPMT is digitized with a 12-bit flash $\mathrm{ADC}$ and read out through VME bus. The timing information is processed and recorded by a TDC. We use a 64-ch, multi-hit TDC developed for ATLAS muon chamber. In order to separate of protons from pions by the energy deposit, the wide dynamic range is required for the readout system. The system is designed to have a good linearity up to 300 p.e., corresponding to $30 \mathrm{MeV}$, while the pedestal width is $2 \mathrm{ADC}$ counts, corresponding to $0.03 \mathrm{MeV}$.

Electron catcher (EC) is located downstream of the tracker. It is used to measure the $\nu_{e}$ contamination in the beam and $\pi^{0}$ production events. The $\mathrm{EC}$ is a calorimeter consists of two planes of 30 (horizontal) and 32 (vertical) modules $\left(4 \times 8 \times 262 \mathrm{~cm}^{3}\right)$ re-used from the CHORUS experiment[6]. Each plane has thickness 5.5 radiation length. A module of $4 \times 4 \mathrm{~cm}^{2}$ cell consists of $1 \mathrm{~mm}$ diameter scintillating fibers in the grooves of lead foils. Lights from both ends of the module are read out by two PMTs. Energy resolution is $14 / \sqrt{E}(\mathrm{GeV}) \%$ with linearity better than $10 \%$. The EC is used for electron and photon identification and the measurement of their energy.

\section{Basic performance}

SciBar is taking data since October 2003. Pedestal, LED and cosmic-ray data are taken simultaneously with beam data for calibration and monitoring. The gain is monitored and corrected by LED, so the energy deposit is measured within a stability better than $1 \%$ by the gain correction. By using cosmic-ray data the attenuation length of WLS fibers are measured as shown in Fig. 2. The average attenuation length is $350 \mathrm{~cm}$ which is consistent with the measurement before installation. Figure 3 shows the pulse height distribution by cosmic-ray muons in one strip. Averaging over all strips, the light yield is 16.5 p.e. $/ \mathrm{cm}$ for minimum ionizating particles at the detector edge close to PMT. The timing resolution is $1.3 \mathrm{nsec}$ with the TQ correction. Neutrino data are taken every 2.2 seconds of beam cycle. The expected number of neutrino interactions is $7 \times 10^{4}$ events in a year. The performance of SciBar is summarized in Table 2. Figure 4 shows the display of a CCQE candidate. We can clearly identify a proton track and a muon by their energy deposit. In one year, $3 \times 10^{4} \mathrm{CCQE}$ events are expected to be observed. SciBar works stably for four months of operation and collects various events of neutrino interactions. 

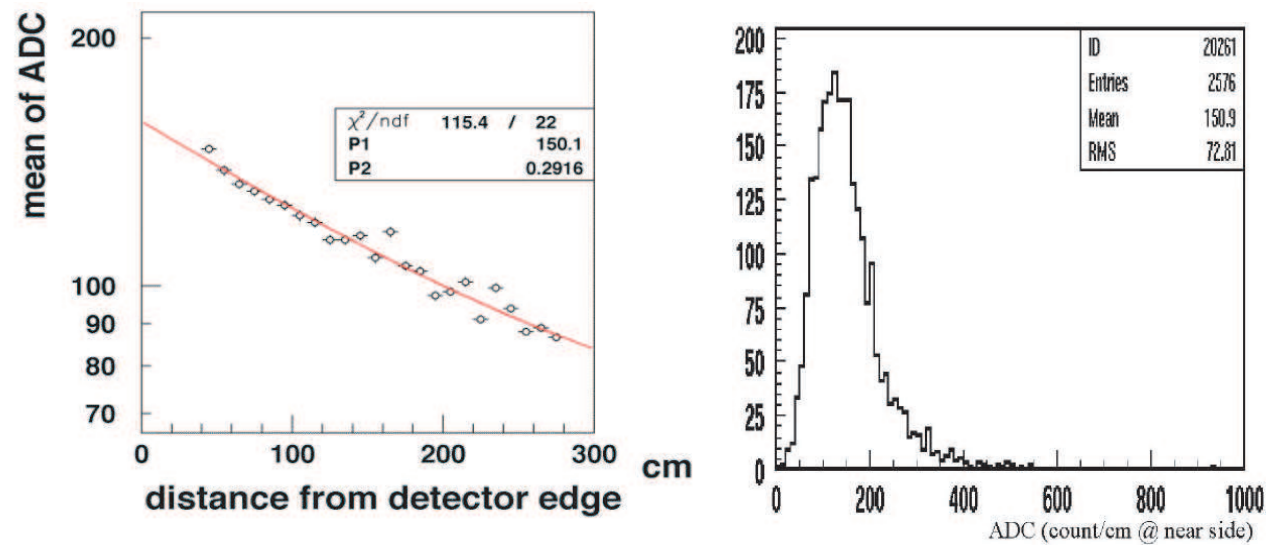

Fig. 2. Attenuation curve of a WLS fiber obtained by the measurement of cosmic-rays.

Fig. 3. Pulse height distribution for a strip by the measurement with cosmic ray. The effect of attenuation in a WLS fiber is corrected.

\begin{tabular}{ll}
\hline \hline Trigger threshold & $0.1 \mathrm{MeV} / \mathrm{strip}$ \\
Attenuation length & $350 \mathrm{~cm}$ \\
Timing resolution & $1.3 \mathrm{nsec}$ \\
Energy resolution & a few $\%(\text { muon })^{*}, \sim 10 \%$ (electron $)^{*}$ \\
Number of neutrino interaction & $7.0 \times 10^{4}$ events $/$ year $\left(3 \times 10^{19} \mathrm{POT}^{* *}\right)^{*}$ \\
$\mathrm{P} / \pi$ misidentification & $5 \%(<1.0 \mathrm{GeV} / \mathrm{c} \text { proton })^{*}$ \\
\hline \hline$*:$ Design value $* *$ : Proton On Target &
\end{tabular}

Table 2

Performance of SciBar

\section{Summary}

The SciBar detector is designed and constructed to measure the neutrino energy spectrum around $1 \mathrm{GeV}$ as the near detector of the $\mathrm{K} 2 \mathrm{~K}$ experiment. , and has been successfully commissioned on schedule. Now SciBar works stably with good performance.

\section{Acknowledgement}

We gratefully acknowledge the assistance of T. Haff and P. Rovegno during the construction of the detector. We would like to express our appreciation for support of M. Taino during the construction work. This work has been undertaken with the support of the Ministry of Education, Culture, Sports, Science and Technology, Government of Japan and its grants for Scientific Research, the Japan Society for Promotion of Science, the U.S. Department of Energy, the Korea Research Foundation, and the Korea Science and Engi- 


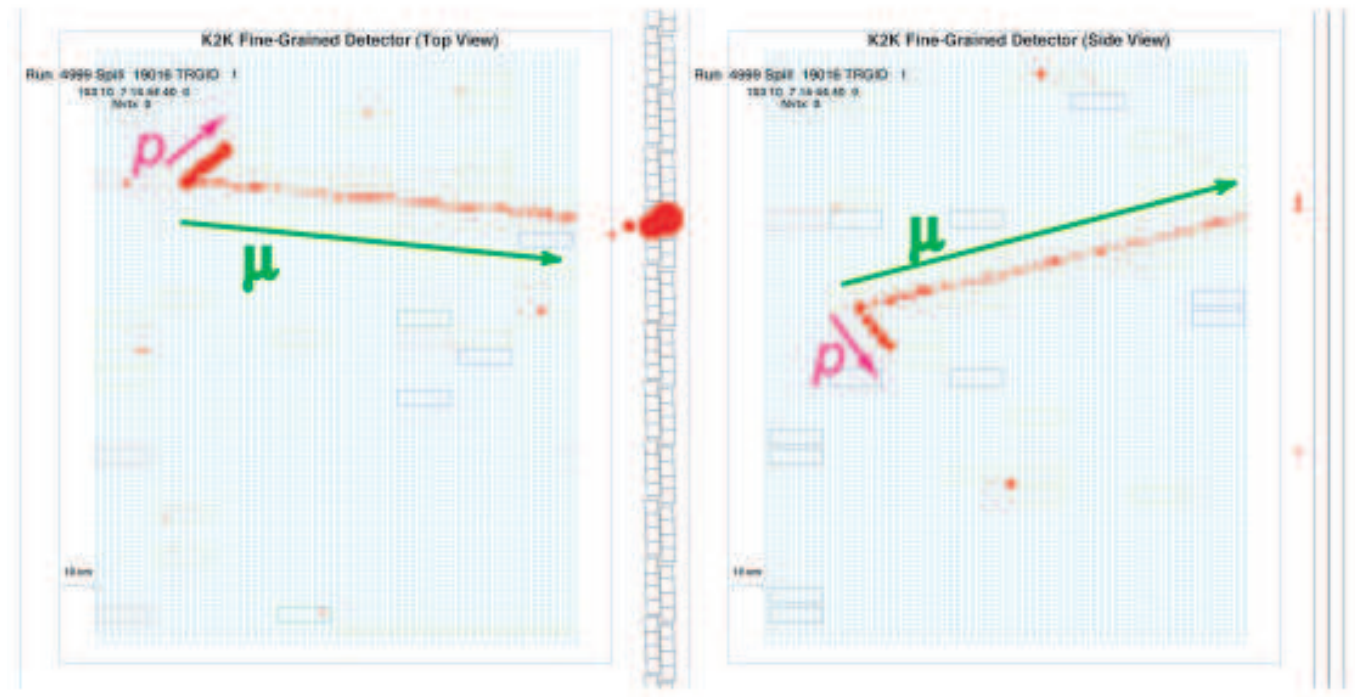

Fig. 4. Event displays of a CCQE candidate in top view (left) and side view (right). Each red circle corresponds to a hit in SciBar. Area of the circle is proportional to the ADC count (i.e. energy deposit) of each channel. Boxes represent TDC hit information by their colors.

neering Foundation. From the spanish institutes, we would like to thank the support of the spanish ministry of science.

\section{References}

[1] The K2K collaboration, MH Ahn et al., Phys. Lett. B511 (2001) 178.

[2] A. Habig for the Super-K Collaboration, 'Atomospheric Neutrino Oscillations in SK-I' proceedings of the 28th International Cosmic Ray Conference(ICRC), Tsukuba, Japan, 2003.

[3] The K2K collaboration, MH Ahn et al., Phys. Rev. Lett. 90 (2003) 041801

[4] M.Yoshida et al., presented at IEEE 2003 Nuclear Science Symposium (NSS).

[5] D.Michael(Caltech. Kellog Lab), Nucl. Phys. Proc. Suppl., 118 (2003) 189.

[6] S.Buontempo et al., Nucl. Phys. Proc. Suppl., 54B (1997) 198 